\title{
Achados ultrassonográficos nos testículos e epidídimos de carneiros deslanados jovens e clinicamente sadios
}

[Ultrasonographic findings in the testis and epididymis of clinically healthy young hair sheep]

\author{
A.K.G. Andrade ${ }^{1}$, A.T. Soares ${ }^{1,2}$, F.Q. Cartaxo $^{2}$, C.E. Peña-Alfaro ${ }^{3}$, M.M. P. Guerra ${ }^{1}$ \\ ${ }^{1}$ Universidade Federal Rural de Pernambuco - Recife, PE \\ ${ }^{2}$ Empresa Estadual de Pesquisa Agropecuária da Paraíba S/A - EMEPA, João Pessoa, PB \\ ${ }^{3}$ Universidade Federal de Campina Grande - Patos, PB
}

\begin{abstract}
RESUMO
Objetivou-se descrever os achados ultrassonográficos nos testículos e epidídimos de carneiros jovens. Análises de desenvolvimento ponderal, mensurações das características biométricas testiculares e exames ultrassonográficos foram realizados dos 140 aos 280 dias de idade, a cada 28 dias. O parênquima testicular apresentou ecogenicidade homogênea (baixa a moderada) que aumentou com a idade. A ecogenicidade e a espessura do mediastino aumentaram com a idade, e a cauda do epidídimo apresentou aspecto hipoecoico em relação ao parênquima testicular. Foram observadas calcificações de grau leve nos testículos de cinco cordeiros. Conclui-se que o exame ultrassonográfico contribui para o monitoramento dos testículos e epidídimos de carneiros.
\end{abstract}

Palavras-chave: ultrassonografia, ecogenicidade, puberdade, ovinos

\begin{abstract}
This study aimed to describe ultrasonographic findings in the testis and epididymis of young sheep. Evaluations of the development of weight, measurements of biometric characteristics of the tests and ultrasound examinations of the tests and epididymis were performed from 140 to 280 days of age, each 28 days. The testicular parenchyma showed homogeneous echogenicity (low to moderate) and increased with the age. The mediastinum echogenicity and thickness increased with age and the epididymis tail showed hypoechoic appearance in relation to the testicular parenchyma. Mild calcification was observed in the testis parenchyma of five lambs. In conclusion, ultrasonographic exams help to monitor testes and epididymis of young hair rams.
\end{abstract}

Keywords: ultrasound, echogenicity, puberty, ovines

\section{INTRODUÇÃO}

O aumento da eficiência reprodutiva de um rebanho constitui uma das principais alternativas para incrementar a sua produtividade. Com este intuito, tem-se verificado crescente introdução de biotecnologias da reprodução, como a ultrassonografia por imagem (US), considerada procedimento simples que pode ser usado para complementar o exame clínico reprodutivo e aumentar a sua precisão.
$\mathrm{Na}$ andrologia, a US é o método preferencial de diagnóstico por imagem, visando avaliar a estrutura anatômica e a determinação da ecogenicidade do parênquima e mediastino testiculares (Chandolia et al., 1997). Este exame pode ser usado como técnica de mensuração das dimensões testiculares (comprimento, largura e altura) in situ, além de ser útil no monitoramento de mudanças progressivas que ocorrem nos testículos (Ahmad, Noakes, 1995). Mesmo com as vantagens da utilização da US no exame andrológico, poucos estudos ultrassonográficos da genitália do macho ovino têm sido descritos,

Recebido em 2 de março de 2011

Aceito em 29 de novembro de 2011

E-mail: annyandrade7@hotmail.com 
especialmente no que se refere à ecogenicidade normal nas diferentes fases do desenvolvimento reprodutivo do macho jovem. Todavia, a ausência de parâmetros normais da morfologia testicular tem sido a principal dificuldade para a utilização da US no diagnóstico andrológico veterinário (Agumbah et al., 1995).

Em virtude da escassez de estudos sobre o aspecto ultrassonográfico normal das estruturas que compõem a genitália externa dos machos ovinos jovens e da importância da utilização da ultrassonografia como exame complementar da avaliação andrológica, bem como do monitoramento de mudanças fisiológicas progressivas que ocorrem nestas estruturas nas diferentes fases do desenvolvimento reprodutivo, objetivou-se descrever os achados ultrassonográficos dos testículos e epidídimos de carneiros deslanados jovens, clinicamente sadios.

\section{MATERIAL E MÉTODOS}

O estudo foi conduzido na Estação Experimental Benjamim Maranhão, pertencente à Empresa Estadual de Pesquisa Agropecuária da Paraíba (EMEPA-PB), localizada no município de Campo de Santana, mesorregião do Agreste Paraibano e microrregião do Curimataú Oriental. Foram utilizados 18 cordeiros machos, clinicamente sadios, mestiços F2 (3/4 Dorper x Santa Inês) e F3 (7/8 Dorper x Santa Inês), incluídos em um único grupo, uma vez que seu genótipo não foi estatisticamente significativo para nenhuma característica em estudo $(\mathrm{P}>0,05)$. Os animais foram criados em sistema de manejo semi-intensivo, tendo acesso à pastagem nativa, silagem de milho e sorgo, concentrado à base de milho $(52,5 \%)$, farelo de trigo $(30 \%)$, farelo de soja $(15 \%)$, cloreto de amônia $(0,5 \%)$, calcário (1\%) e sal mineral (1\%); além de água e sal mineral ad libitum. A suplementação mineral fornecida apresentou a seguinte composição por quilograma: Na 147g; Ca 120g; P 87g; S 18g; Zn $3.800 \mathrm{mg}$; Fe 3500mg; Mn 1.300mg; Fl $870 \mathrm{mg}$; $\mathrm{Cu} 590 \mathrm{mg}$; Mo 300mg; I 80mg; Co 40mg; Cr 20mg; Se 15mg.

Objetivando-se detectar enfermidades que poderiam interferir no potencial reprodutivo dos animais, eles foram submetidos à avaliação clínica geral e morfológica dos órgãos genitais externos (inspeção e palpação do escroto, testículos, epidídimos, cordões espermáticos, pênis e prepúcio), como critério de seleção, antes de cada momento de avaliação.

Em intervalos de 28 dias, dos 140 aos 280 dias de idade, foram realizadas avaliações do desenvolvimento ponderal, mensurações das características biométricas dos testículos (circunferência escrotal, largura e comprimento) e exames ultrassonográficos dos testículos e epidídimos. Por meio de colheitas periódicas de sêmen, no período que correspondeu ao desbridamento do pênis ao prepúcio até o surgimento de ejaculados apresentando espermatozoides com taxa de motilidade acima de $10,0 \%$ e, no mínimo, 50×10 espermatozoides/mL (Wolf et al., 1965), estabeleceu-se o momento em que os cordeiros apresentaram maturidade reprodutiva. As colheitas de sêmen foram realizadas por eletroejaculação e as amostras avaliadas quanto à presença de turbilhonamento, motilidade, vigor e concentração espermáticas (Mies Filho, 1987).

O perímetro escrotal (PE) foi medido utilizandose uma fita milimetrada, posicionada na porção de maior diâmetro do escroto. Com o auxílio de um paquímetro, foram mensudados $\mathrm{o}$ comprimento e a largura testiculares (CT e LT, respectivamente). Para medir o CT, consideraram-se os testículos, excluindo a cauda dos epidídimos no sentido dorsoventral. A LT foi aferida na porção média do testículo no sentido lateromedial. Os resultados foram expressos em centímetros $(\mathrm{cm})$. A equação matemática do prolato esferoide proposta por Bayley et al. (1998): VOL $=2 \times\left[4 / 3 \times \pi \times(\mathrm{LT} / 2)^{2} \times(\mathrm{CT} / 2)\right]$ foi utilizada para determinação do volume testicular, o qual foi expresso em centímetro cúbico $\left(\mathrm{cm}^{3}\right)$ e representou a média dos dois testículos.

As avaliações ultrassonográficas dos testículos foram realizadas em aparelho Pie Medical (modelo Falco 100), utilizando-se transdutor de arranjo linear com frequência de $8,0 \mathrm{MHz}$, e as imagens documentadas em impressora (Sony, São Paulo, Brasil). Com a finalidade de minimizar as variáveis que pudessem interferir nos resultados, as avaliaçoes foram realizadas por um único profissional e o aparelho de ultrassom foi ajustado sempre da mesma maneira ao início das mensurações. Após tricotomia da região escrotal, gel de contato foi aplicado 
diretamente sobre a superfície a ser examinada, prosseguindo-se com varreduras nos planos sagital, transversal, frontal e oblíquo dos testículos direito e esquerdo de cada animal, avaliando-se, desse modo, a ecotextura do parênquima e mediastino testiculares, além da cauda do epidídimo e do plexo pampiniforme. $\mathrm{O}$ plano de varredura oblíquo foi utilizado apenas para avaliação da cauda do epidídimo. Em virtude da utilização de um transdutor de arranjo linear, que dificultou o contato entre ele e a cabeça e corpo do epidídimo, estas avaliações foram preteridas. Além disso, foram realizadas aferições ultrassonográficas da espessura do mediastino (plano frontal) e largura testicular (plano transversal), em ambos os testículos. As medidas de largura testicular mensuradas por ultrassom foram comparadas com as medidas de largura testicular aferidas com paquímetro. Para ambas as medidas, foi adotado o centímetro como unidade.

Estatísticas descritivas foram efetuadas em várias possibilidades de agregação dos dados referentes à ecogenicidade dos testículos e estruturas relacionadas. Para análise estatística das características do sêmen e da biometria testicular, foi utilizado o programa computacional Statistical Analysis System (SAS, 2000), realizando-se análise de variância (ANOVA) e as diferenças entre as médias avaliadas pelo teste de comparação múltipla Tukey-Kramer, a 5\% de significância. As medidas de largura dos testículos obtidas com paquímetro foram comparadas com as aferidas nas imagens ultrassonográficas em planos transversais, e suas médias analisadas pelo teste $\mathrm{Z}$ a $1 \%$ de significância. A espessura de mediastino testicular $(\mathrm{cm})$, perímetro escrotal $(\mathrm{cm})$ e volume testicular $\left(\mathrm{cm}^{3}\right)$ foi analisada sob a forma de equações de regressões lineares em função da idade.

\section{RESULTADOS E DISCUSSÃO}

A análise do sêmen dos cordeiros (Tab. 1) evidenciou aumento $(\mathrm{P}<0,05)$ nas médias de turbilhonamento, motilidade progressiva e concentração espermática, bem como redução $(\mathrm{P}<0,05)$ na porcentagem de defeitos morfológicos dos espermatozoides com o aumento da idade dos animais (dos 140 aos 280 dias). O elevado desvio-padrão observado pode ser explicado pelas diferentes fases de maturidade sexual dos animais, apesar de estes fazerem parte de um mesmo grupo etário, além da diferença de peso corporal, uma vez que este parâmetro possui maior correlação com a puberdade do que a idade do animal (Skinner $e t$ al., 1968). O resultado da avaliação espermática mostrou que os cordeiros mestiços apresentam precocidade sexual em relação aos animais puros da raça Santa Inês (dados não publicados), provavelmente devido ao efeito heterose, que possibilita o rápido desenvolvimento corporal.

A avaliação da ecotextura testicular dos cordeiros evidenciou parênquima testicular (PT) homogêneo, com ecogenicidade variando de baixa (Fig. 1a e 1b) a moderada (Fig. 1c e 1d), independentemente do testículo (direito ou esquerdo) e do plano de varredura usado, tendo ela aumentado em proporção direta com a idade dos animais, corroborando com Cartee et al. (1990), Ahmad et al. (1991), Gouletsou et al. (2003) e Andrade Moura et al. (2008). Contudo, apesar da similaridade entre os trabalhos, verifica-se que a nomenclatura utilizada para descrever o aspecto ultrassonográfico do PT apresenta marcantes variações entre autores, havendo, portanto, a necessidade de padronização desta, a fim de facilitar a realização de novos estudos, assim como o estabelecimento de parâmetros de normalidade para esta espécie.

Quando comparados os testículos de cordeiros pré-púberes aos dos cordeiros da mesma idade apresentando maturidade reprodutiva (Tab. 1), verificou-se predominância das imagens com baixa ecogenicidade nos primeiros (140 dias) e moderada ecogenicidade nos segundos (a partir de 168 dias de idade). O aumento da ecogenicidade do PT em proporção direta com a idade dos animais assemelha-se ao obervado por Hamm e Fobbe (1995), Chandolia et al. (1997) e Brito et al. (2004), estando relacionado às mudanças anatômicas dos túbulos seminíferos, os quais se tornam mais longos e retorcidos, aumentam de diâmetro e formam o lúmen (Hamm, Fobbe, 1995), devido ao aumento da espessura da membrana basal, evidenciando a possibilidade do uso da US no monitoramento das mudanças progressivas que ocorrem nos testículos (Ahmad e Noakes, 1995). 
Tabela 1. Média ( \pm desvio-padrão) dos parâmetros seminais (turbilhonamento, motilidade progressiva e vigor) e da frequência da ecogenicidade do parênquima testicular e do mediastino testicular (plano frontal) de carneiros mestiços (Dorper x Santa Inês) na fase peripuberal

\begin{tabular}{|c|c|c|c|c|c|c|c|c|c|}
\hline \multirow{2}{*}{$\begin{array}{l}\text { Idade } \\
\text { (dias) }\end{array}$} & \multirow{2}{*}{$\begin{array}{l}\text { Turb. } \\
(0-5)\end{array}$} & \multirow{2}{*}{$\begin{array}{l}\text { MP } \\
(\%)\end{array}$} & \multirow{2}{*}{$\begin{array}{l}\text { Vigor } \\
(0-5)\end{array}$} & \multirow{2}{*}{$\begin{array}{l}\text { Concentração } \\
\text { (x109) }\end{array}$} & \multirow{2}{*}{$\begin{array}{c}\text { Defeitos } \\
\text { totais } \\
(\%)\end{array}$} & \multicolumn{2}{|c|}{ Parênquima testicular (\%) } & \multicolumn{2}{|c|}{ Mediastino testicular (\%) } \\
\hline & & & & & & Direito & Esquerdo & Direito & Esquerdo \\
\hline \multirow[t]{4}{*}{140} & $1,10 \pm$ & $31,00 \pm$ & $2,60 \pm$ & $0,82 \pm$ & $15,89 \pm$ & $55,5 \mathrm{BE}$ & $55,5 \mathrm{BE}$ & $61,1 \mathrm{ME}$ & $61,1 \mathrm{ME}$ \\
\hline & $0,88 \mathrm{a}$ & $15,24 \mathrm{a}$ & 1,26 & $0,43 a$ & $3,67 \mathrm{a}$ & & & & \\
\hline & & & & & & 45,5ME & 45,5ME & $22,2 \mathrm{AE}$ & $22,2 \mathrm{AE}$ \\
\hline & & & & & & & & $16,7 \mathrm{DIF}$ & $16,7 \mathrm{DIF}$ \\
\hline \multirow[t]{4}{*}{168} & $2,00 \pm$ & $48,46 \pm$ & $3,00 \pm$ & $1,34 \pm$ & $13,68 \pm$ & $38,9 \mathrm{BE}$ & $38,9 \mathrm{BE}$ & $55,5 \mathrm{ME}$ & $55,5 \mathrm{ME}$ \\
\hline & $1,15 \mathrm{ab}$ & $18,64 \mathrm{ab}$ & 0,58 & $0,77 a b$ & $3,06 a b$ & & & & \\
\hline & & & & & & $61,1 \mathrm{ME}$ & $61,1 \mathrm{ME}$ & $27,8 \mathrm{AE}$ & $27,8 \mathrm{AE}$ \\
\hline & & & & & & & & $16,7 \mathrm{DIF}$ & $16,7 \mathrm{DIF}$ \\
\hline \multirow[t]{3}{*}{196} & $2,50 \pm$ & $54,29 \pm$ & $3,21 \pm$ & $1,88 \pm$ & $11,21 \pm$ & $33,3 \mathrm{BE}$ & $33,3 \mathrm{BE}$ & $55,5 \mathrm{ME}$ & $55,5 \mathrm{ME}$ \\
\hline & $1,02 b c$ & $20,65 b c$ & 0,80 & $1,09 a b$ & $3,96 b c$ & & & & \\
\hline & & & & & & $66,7 \mathrm{ME}$ & $66,7 \mathrm{ME}$ & $\begin{array}{l}27,8 \mathrm{AE} \\
167 \mathrm{DIF}\end{array}$ & $\begin{array}{l}27,8 \mathrm{AE} \\
167 \mathrm{DIF}\end{array}$ \\
\hline \multirow[t]{3}{*}{224} & $3,11 \pm$ & $64,44 \pm$ & $3,44 \pm$ & $2,04 \pm$ & $10,94 \pm$ & $16,7 \mathrm{BE}$ & $16,7 \mathrm{BE}$ & $50,0 \mathrm{ME}$ & $50,0 \mathrm{ME}$ \\
\hline & $1,37 b c$ & $20,07 \mathrm{bcd}$ & 1,04 & $1,32 b$ & $3,96 \mathrm{c}$ & $833 \mathrm{MF}$ & $833 \mathrm{MF}$ & & \\
\hline & & & & & & $83,3 \mathrm{MIE}$ & $83,3 \mathrm{MIE}$ & $\begin{array}{l}33,3 \mathrm{AE} \\
16,7 \mathrm{DIF}\end{array}$ & $\begin{array}{l}33,3 \mathrm{AE} \\
16,7 \mathrm{DIF}\end{array}$ \\
\hline \multirow[t]{3}{*}{252} & $3,33 \pm$ & $68,89 \pm$ & $3,50 \pm$ & $2,32 \pm$ & $9,28 \pm$ & $11,1 \mathrm{BE}$ & $11,1 \mathrm{BE}$ & $50,0 \mathrm{ME}$ & $50,0 \mathrm{ME}$ \\
\hline & $1,03 \mathrm{c}$ & $12,68 \mathrm{~cd}$ & 0,92 & $1,06 \mathrm{~b}$ & $4,42 \mathrm{c}$ & & & & \\
\hline & & & & & & $88,9 \mathrm{ME}$ & $88,9 \mathrm{ME}$ & $\begin{array}{l}38,8 \mathrm{AE} \\
112 \mathrm{DIF}\end{array}$ & $38,8 \mathrm{AE}$ \\
\hline \multirow[t]{3}{*}{280} & $3,44 \pm$ & $72,80 \pm$ & $3,61 \pm$ & $2,34 \pm$ & $8,78 \pm$ & $11,1 \mathrm{BE}$ & $11,1 \mathrm{BE}$ & $44,4 \mathrm{ME}$ & $44,4 \mathrm{ME}$ \\
\hline & $0,86 \mathrm{c}$ & $15,30 \mathrm{~d}$ & 0,85 & $1,14 \mathrm{~b}$ & $2,67 \mathrm{c}$ & & & & \\
\hline & & & & & & $88,9 \mathrm{ME}$ & $88,9 \mathrm{ME}$ & $44,4 \mathrm{AE}$ & $44,4 \mathrm{AE}$ \\
\hline
\end{tabular}

Turb. = turbilhonamento; $\mathrm{MP}=$ motilidade progressiva; $\mathrm{AE}=$ alta ecogenicidade $; \mathrm{ME}=$ média ecogenicidade; $\mathrm{BE}=$ baixa ecogenicidade; $\mathrm{DIF}=$ difuso. Letras diferentes na mesma coluna indicam diferença estatística $(\mathrm{P}<0,05)$.

Segundo Gipson et al. (1985), com a identificação do mediastino testicular (MT) e uma clara distinção entre este e o parênquima, é possível medir o diâmetro dos testículos, com o congelamento da imagem e a utilização do cursor manual eletrônico, tendo como referência a túnica albugínea. Neste estudo, o MT (Tab. 1) avaliado em plano frontal foi visibilizado em $100 \%$ dos animais e caracterizado como uma linha hiperecoica de espessura e ecogenicidade variáveis no centro do PT. Todavia, a análise em plano transversal evidenciou um ponto hiperecoico também no centro do PT. Quanto à ecotextura, o MT foi classificado em moderadamente ecogênico (Fig. 1e e 1h), altamente ecogênico (Fig. 1f e 1i) e difuso (Fig. $1 \mathrm{~g})$, verificando-se aumento da ecogenicidade e da espessura em proporção direta com a idade dos animais. Este resultado assemelha-se aos relatados por Ahmad et al. (1991), Gouletsou et al. (2003) e Andrade Moura et al. (2008). Gouletsou et al. (2003) descreveram que o MT, uma massa de tecido fibroso contendo numerosos tubos finos na parte central dos testículos, foi visibilizado em $87 \%$ dos animais e que a frequência de alta ecogenicidade aumenta com a idade animal, o que foi também observado neste estudo e que pode ser atribuído às mudanças anatômicas que ocorrem nos túbulos seminíferos (Hamm e Fobbe, 1995).

O exame de US realizado aos 224 dias mostrou, no PT direito e no esquerdo de dois dos 18 cordeiros avaliados, calcificações de grau leve, caracterizadas como imagens multifocais hiperecoicas. Aos 252 dias, mais três animais apresentaram tais achados, os quais permaneceram até a última avaliação (Fig. $2 \mathrm{a}$ e 2b), evidenciando aumento progressivo do número de cordeiros com quadro semelhante, bem como do número de focos em cada testículo em proporção direta com a idade dos animais. Estes achados demonstram a necessidade de estudos mais aprofundados, uma vez que tais calcificações foram descritas, geralmente, associadas a processos patológicos (Hamm, Fobbe, 1995; Ganem et al., 1999; Sartori et al., 2002), e, no presente estudo, assim como nos relatos de Andrade Moura et al. (2008), não foram associadas à alteração clínica, mas ao avanço da idade dos animais. 
Tabela 2. Média ( \pm desvio-padrão) de perímetro escrotal (PE), volume testicular (VT), largura testicular aferida com paquímetro (LPQ), largura testicular aferida com ultrassom (LUS) e espessura do mediastino (MT), de carneiros mestiços (Dorper x Santa Inês) na fase peripuberal

\begin{tabular}{|c|c|c|c|c|c|c|c|c|}
\hline \multirow{2}{*}{$\begin{array}{l}\text { Idade } \\
\text { (dias) }\end{array}$} & \multirow{2}{*}{$\begin{array}{l}\text { PE } \\
(\mathrm{cm})\end{array}$} & \multirow{2}{*}{$\begin{array}{c}\mathrm{VT} \\
\left(\mathrm{cm}^{3)}\right.\end{array}$} & \multicolumn{2}{|c|}{ LPQ (cm) } & \multicolumn{2}{|c|}{ LUS (cm) } & \multicolumn{2}{|c|}{ MT $(\mathrm{cm})$} \\
\hline & & & $\mathrm{D}$ & $E$ & $\mathrm{D}$ & $\mathrm{E}$ & $\mathrm{D}$ & $E$ \\
\hline \multirow{2}{*}{140} & $20,94 \pm$ & $100,70 \pm$ & $4,16 \pm$ & $4,17 \pm$ & $3,87 \pm$ & $3,89 \pm 0,7$ & $0,19 \pm 0$ & $0,19 \pm 0$ \\
\hline & $5,77 \mathrm{a}$ & $55,49 \mathrm{a}$ & $0,78 \mathrm{a}$ & $0,76 \mathrm{a}$ & 0,80 & 7 &, $04 a$ & $05 \mathrm{a}$ \\
\hline \multirow{2}{*}{168} & $23,11 \pm$ & $150,70 \pm$ & $4,54 \pm$ & $4,54 \pm 0$ & $4,12 \pm$ & $4,11 \pm$ & $0,22 \pm$ & $0,22 \pm$ \\
\hline & $5,25 \mathrm{ab}$ & $76,60 \mathrm{ab}$ & $0,80 \mathrm{ab}$ & $75 \mathrm{ab}$ & 0,75 & 0,71 & $0,04 \mathrm{ab}$ & $0,04 \mathrm{ab}$ \\
\hline \multirow{2}{*}{196} & $25,92 \pm$ & $191,52 \pm$ & $5,04 \pm$ & $5,01 \pm$ & $4,25 \pm$ & $4,27 \pm$ & $0,23 \pm$ & $0,24 \pm$ \\
\hline & $3,37 \mathrm{bc}$ & $76,75 b c$ & $0,65 \mathrm{bc}$ & $0,65 \mathrm{bc}$ & 0,65 & 0,62 & $0,05 \mathrm{ab}$ & $0,05 \mathrm{ab}$ \\
\hline \multirow{2}{*}{224} & $27,19 \pm$ & $222,54 \pm$ & $5,24 \pm$ & $5,27 \pm$ & $4,35 \pm$ & $4,34 \pm$ & $0,24 \pm$ & $0,24 \pm$ \\
\hline & $2,78 \mathrm{c}$ & $64,75 c$ & $0,51 \mathrm{c}$ & $0,50 \mathrm{c}$ & 0,51 & 0,49 & $0,05 \mathrm{ab}$ & $0,04 \mathrm{ab}$ \\
\hline \multirow{2}{*}{252} & $28,53 \pm$ & $240,90 \pm$ & $5,31 \pm$ & $5,37 \pm$ & $4,40 \pm$ & $4,39 \pm$ & $0,25 \pm$ & $0,26 \pm$ \\
\hline & $2,56 \mathrm{c}$ & $69,90 \mathrm{c}$ & $0,48 c$ & $0,49 c$ & 0,43 & 0,46 & $0,07 \mathrm{ab}$ & $0,05 b$ \\
\hline \multirow{2}{*}{280} & $29,67 \pm$ & $256,60 \pm$ & $5,36 \pm$ & $5,34 \pm$ & $4,48 \pm$ & $4,47 \pm$ & $0,26 \pm$ & $0,26 \pm$ \\
\hline & $2,20 \mathrm{c}$ & $66,00 \mathrm{c}$ & $0,43 c$ & $0,43 c$ & 0,36 & 0,38 & $0,06 \mathrm{~b}$ & $0,05 \mathrm{~b}$ \\
\hline
\end{tabular}

$\mathrm{D}=$ direito; $\mathrm{E}=$ esquerdo. Letras diferentes na mesma coluna indicam diferença estatística $(\mathrm{P}<0,05)$.

A aferição do comprimento do parênquima testicular em animais mais velhos foi uma limitação encontrada com o uso da sonda linear, a partir do momento em que o comprimento dos testículos ultrapassou o do transdutor (Bayley et al., 1998), assim como a cabeça e o corpo do epidídimo (Pechman e Eilts, 1987; Ahmad et al., 1991; Andrade Moura et al., 2008). Todavia, o comprimento total da cabeça do epidídimo também pode ter sido mascarado pelo plexo pampiniforme na sua porção superior (Gouletsou et al., 2003). Desta forma, ressalta-se que estudos semelhantes devem ser realizados com uso de transdutores convexos e microconvexos, que produzem imagem setorial, com campo de visão mais amplo do que os lineares, necessitando de uma menor área de contato deste com a pele do animal (Nyland et al., 2004).

A cauda do epidídimo foi claramente visibilizada em $100 \%$ dos animais, apresentando-se hipoecoico em relação ao PT (Fig. 2c), corroborando com relatos anteriores (Ahmad et al., 1991; Gouletsou et al., 2003). O septo escrotal (Fig. 2d) foi observado na ultrassonografia como uma linha hiperecogênica entre os testículos. As túnicas testiculares (Fig. 2e) não puderam ser identificadas separadamente, uma vez que isto só ocorre na presença de líquido entre elas, tendo sido observadas circundando o testículo e separando o seu parênquima do escroto. A camada de fluido normal entre as túnicas nunca excede dois milímetros de espessura e somente quando excede esse valor pode ser considerada patológica (Pechman e Eilts, 1987; Gouletsou et al., 2003).

O plexo pampiniforme (Fig. 2f) foi claramente visibilizado nos planos frontal e transversal, principalmente com o aumento da idade dos animais, quando os testículos se tornaram mais pendulosos, apresentando ecogenicidade reduzida em relação ao PT, sendo caracterizado por numerosas estruturas tubulares anecoicas (Pechman e Eilts, 1987; Ahmad et al., 1991; Gouletsou et al., 2003).

A média de largura testicular aferida com paquímetro (LPQ) dos 140 aos 280 dias de idade foi de $4,95 \mathrm{~cm}$, maior do que aquela obtida ao ultrassom $(4,24 \mathrm{~cm})$. No entanto, segundo Bayley et al. (1998), a largura de testículos bovinos aferida com ultrassom apresenta maior correlação com a largura real, quando comparada àquela obtida com o paquímetro, uma vez que, ao ultrassom, somente o parênquima testicular é aferido, ao contrário do paquímetro, em que, nos valores obtidos, estão incluídos o corpo do epidídimo, o escroto e as túnicas testiculares. Segundo estes autores, o volume testicular obtido com paquímetro apresenta correlação maior com o volume real em relação ao ultrassom, o que pode ser explicado pela dificuldade de se avaliar o comprimento testicular ao ultrassom em animais mais velhos, conforme foi evidenciado neste estudo. 

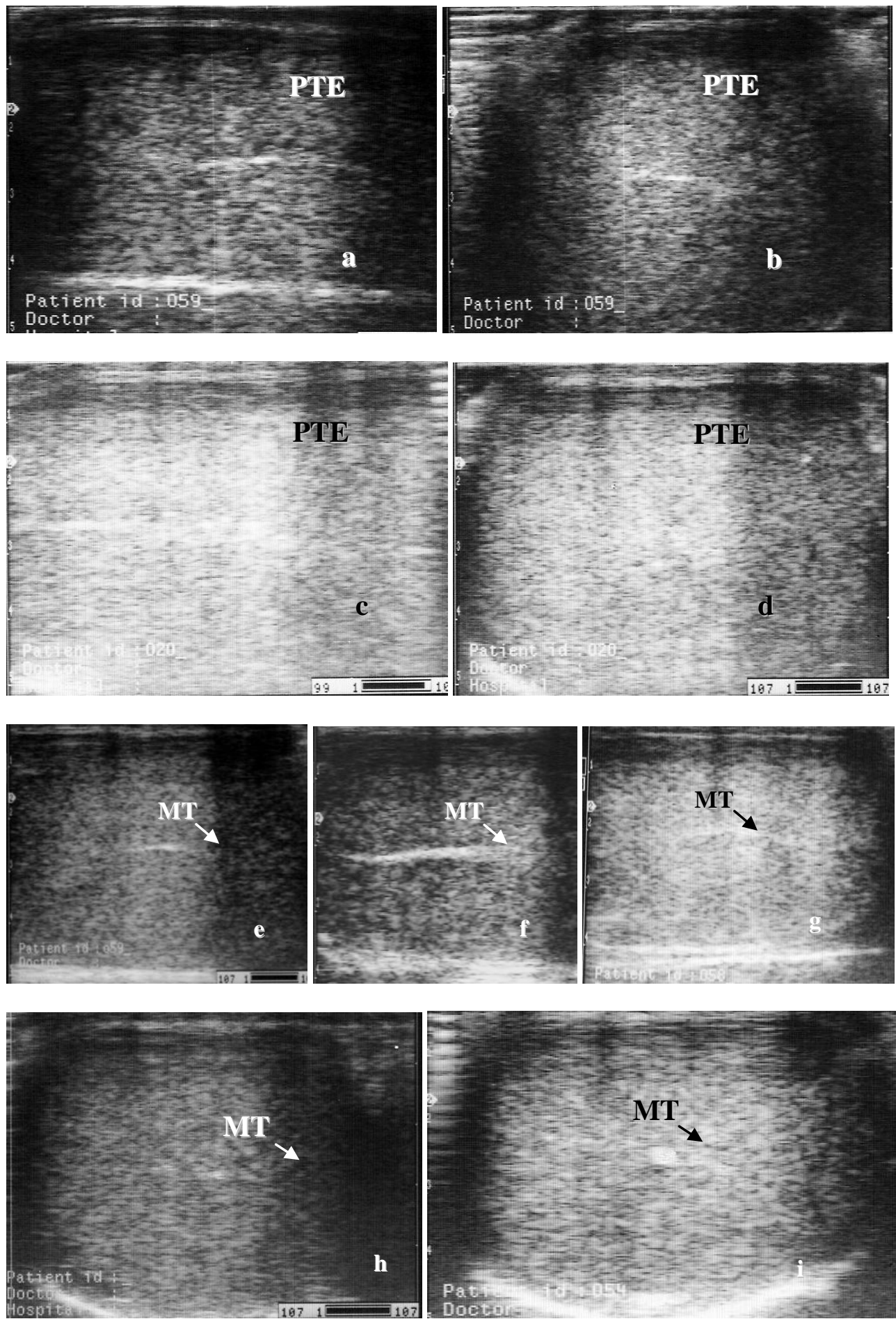

Figura 1. Imagens ultrassonográficas de testículos de ovinos mestiços clinicamente sadios, evidenciando: baixa ecogenicidade do parênquima testicular esquerdo (PTE), aos 140 dias de idade - plano sagital (a) e transverso (b); moderada ecogenicidade do PTE, aos 252 dias de idade - plano sagital (c) e transverso (d); mediastinos testiculares (MT) dos tipos moderadamente ecogênico (e), altamente ecogênico (f) e difuso (g), aos 224 dias de idade - plano frontal; mediastinos testiculares (MT) dos tipos moderadamente ecogênico (h) e altamente ecogênico (i), aos 224 dias de idade - plano transversal. 

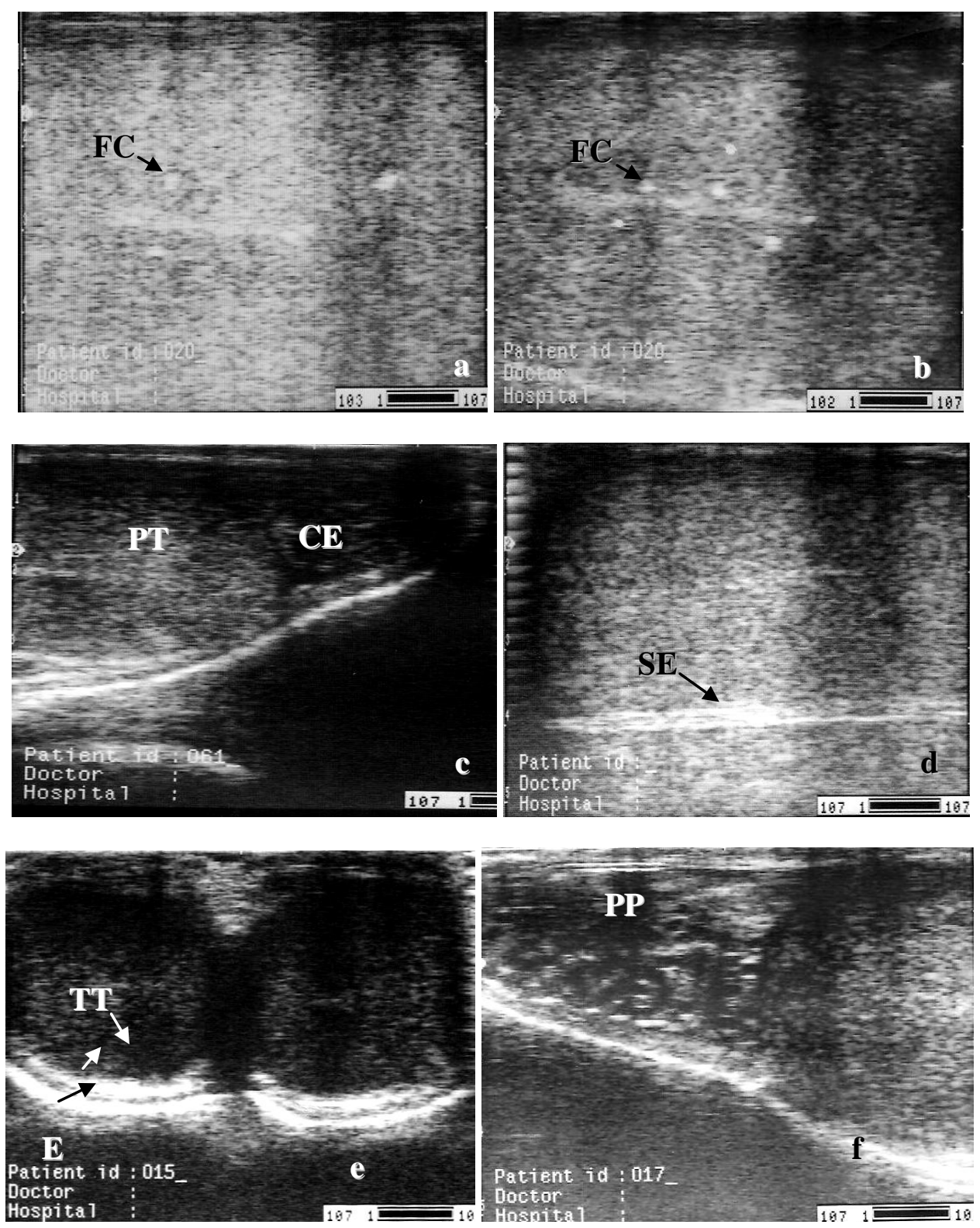

Figura 2. a, b = Imagem ultrassonográfica do testículo esquerdo de ovino mestiço, clinicamente sadio aos 224 (a) e 280 (b) dias de idade, apresentando focos de calcificação (FC) testicular - plano sagital. c, d, e, $\mathrm{f}=$ Imagens ultrassonográficas de testículos de ovinos mestiços, clinicamente sadios, evidenciando: parêquima testicular (PT) e cauda do epidídimo (CE), aos 250 dias de idade (c); septo escrotal (SE), aos 168 dias de idade - plano frontal (d); túnicas testiculares (TT) e escroto (E), aos 140 dias - plano transversal (e) e plexo pampinoforme (PP), aos 224 dias de idade - plano sagital (f).

No entanto, ressalta-se que a análise biométrica do desenvolvimento testicular tem grande importância, estando significativamente correlacionada com a futura capacidade reprodutiva do animal (Mukasa-Mugerwa, Azaz, 1992). Neste estudo, observou-se que o perímetro escrotal (PE), o volume testicular (VT) e a espessura do mediastino testicular (MT) apresentaram aumento em proporção direta com a idade dos animais, cujas equações de regressão linear obtidas foram, respectivamente, $\mathrm{y}=12,78$ $+0,06 x ; y=-37,84+1,10 x ; y=0,13+0,0005 x$. A análise de VT apresentou crescimento mais acelerado dos 140 aos 224 dias, coincidindo com 
o momento em que os indivíduos apresentaram maturidade reprodutiva, o que reforça os relatos de Unanian et al. (2000) de que este parâmetro deve ser usado na seleção de reprodutores, além do perímetro escrotal, uma vez que carneiros com maior perímetro escrotal realizam coberturas mais cedo e suas filhas atingem a puberdade mais precoce e com maior taxa de ovulação (Ott, Memon, 1980).

As médias da espessura do mediastino variaram de $0,19 \mathrm{~mm}$ a $0,26 \mathrm{~mm}$, dos 140 aos 280 dias de idade, havendo grande variação entre cordeiros na mesma idade, provavelmente devido às diferentes fases de maturidade reprodutiva nas quais eles se encontravam. De acordo com Sisson (1996), no ruminante adulto essa faixa de tecido conjuntivo mede, aproximadamente, cinco milímetros. Vale ressaltar que para as três variáveis avaliadas (perímetro escrotal, volume testicular e espessura do mediastino), a fase de crescimento menos acentuado foi dos 250 aos 280 dias, período em que todos os animais já haviam atingido a puberdade, corroborando com Chandolia et al. (1997), ao relatarem que a taxa de crescimento testicular dos ovinos é lenta nos dois primeiros meses de idade, acelerando com o início da espermatogênese e reduzindo novamente após a puberdade.

O aspecto ultrassonográfico dos testículos e epidídimos de ovinos clinicamente sadios já havia sido estudado (Cartee et al., 1990; Gouletsou et al., 2003; Andrade Moura et al., 2008, Jucá et al., 2009). Contudo, apenas Ahmad et al. (1991) acompanharam ultrassonograficamente o desenvolvimento testicular de ovinos com idades próximas a dos animais deste experimento, o que ressalta a escassez de trabalhos na área.

\section{CONCLUSÃO}

Os resultados possibilitam concluir que a ultrassonografia por imagem é adequada na avaliação morfofisiológica da genitália externa de ovinos, contribuindo para a identificação e o monitoramento das mudanças fisiológicas progressivas que ocorrem nos testículos e epidídimos de ovinos deslanados jovens, clinicamente sadios.

\section{AGRADECIMENTOS}

À Fundação de Amparo à Ciência e Tecnologia do Estado de Pernambuco (FACEPE), à Empresa Estadual de Pesquisa Agropecuária da Paraíba S/A (EMEPA).

\section{REFERÊNCIAS}

AGUMBAH, G.J.O.; ODIANO, G.O.; TYREL, D. et al. Ultrasonography to diagnose testicular lesions in infertile Angora buck. Irish Vet. J., v.48, p.416-418, 1995.

AHMAD, N.; NOAKES, D.E.; SUBANDRIO, A.L. B-mode real time ultrasonographic imaging of the testis and epididymis of sheep and goats. Vet. Rec., v.12, p.491-496, 1991.

AHMAD, N.; NOAKES, D.E. A clinical and ultrasonographic study of induced testicular and epididymal lesions in goats and a ram. Anim. Reprod. Sci., v.39, p.35-48, 1995.

ANDRADE MOURA, J.C.; JUCÁ, A.F.; GUSMÃO, A.L. et al. Ecotextura testicular do carneiro Santa Inês. Hora Vet. , v.27, p.19-22, 2008.

BAYLEY, T.L.; HUDSO, R.; POWE, T. et al. Caliper ultrasonographic measurements of bovine testicles and a mathematical formula for determining testicular volume and weight in vivo. Theriogenology, v.49, p.581-594, 1998.

BRITO, L.F; SILVA, A.E.; UNANIAN, M.M. et al. Sexual development in early an late maturing Bos indicus and Bos indicus $x$ Bos taurus crossbred bulls in Brasil. Theriogenology, v.62, p.1198-1217, 2004.

CARTEE, R.E.; RUMPH, P.F.; ABUZAID, S. et al. Ultrasonographic examination and measurement of ram testicles. Theriogenology, v.33, p.867-875, 1990.

CHANDOLIA, R.K.; BARTLEWSKI, P.M.; OMEKE, B.C. et al. Ultrasonography of the developing reproductive tract in ram lambs effects of a GnRH agonist. Theriogenology, v.48, p.99-117, 1997.

GANEM, J. P.; WORKMAN, K.R.; SHABAN, S.F. Testicular microlithiasis associated with testicular pathology. Adult Urol., v.53, p.209-213, 1999.

GIPSON, T.A.; VOGT, D.W.; MASSEY J.W. et al. Association of scrotal circumference with semen traits in young beef bulls of different breeds. Theriogenology, v.24, p.217-225, 1985.

GOULETSOU, P.G.; AMIRIDIS, G.S.; CRIPPS, P.J. et al. Ultrasonographic appearance of clinically healthy testicles and epididymes of rams. Theriogenology, v.59, p.1959-1972, 2003. 
HAMM, B.; FOBBE, F. Maturation of the testis: ultrasound evaluation. Ultrasound Med. Biol., v.21, p.143-147, 1995.

JUCÁ, A.F.; ANDRADE MOURA, J.C.; GUSMÃO, A.L. et al. Avaliação ultrassonográfica dos testículos e das glândulas sexuais anexas de carneiros Santa Inês. Cienc. Anim. Bras., v.10, p.650-659, 2009.

MIES FILHO, A. Inseminação Artificial, 6a ed., Porto Alegre: Sulina, v.2, 1987, 750p.

MUKASA-MUGERWA, E.; EZAZ, Z. Relationship of testicular growth and size to age, body weight and onset of puberty in Menz ram lambs. Theriogenology, v.38, p.979-988, 1992.

NYLAND, T.G.; MATTOON, J.S.; HERRGESELL, E.J. et al. Princípios físicos, instrumentação e segurança do diagnóstico por ultrassom. In: NYLAND, T. G.; MATTOON, J.S. Ultrassom diagnóstico em pequenos animais. 2.ed., São Paulo: Roca, 2004. p.1-19.

OTT, R.S.; MEMON, M.A Breeding soundness examinations of rams and bucks: a review. Theriogenology, v.13, p.155-164,1980.

PECHMAN, R.D.; EILTS, B.E. B-mode ultrasonography of the bull testicle. Theriogenology, $\mathrm{v}$. 27, p. 431-441, 1987.
SARTORI, R.; PRESTES, N.C.; CANAVESSI, A.M.O. et al. Avaliações ultrassonográfica, macroscópica e histológica da biopsia testicular em ovinos. Arq. Bras. Med. Vet. Zootec., v.54, p.1-12, 2002.

SAS INSTITUTE. System for information. Versão 8.0. Cary:SAS Institute, 2000.CD-ROM.

SKINNER, J.D.; BOOTH, W.D.; ROWSON, L.E.A. et al. The post-natal development of the reproductive tract of the Suffolk ram, and changes in the gonadotrophin content of the pituitary. J. Reprod. Fertil., v.16, p.463 - 477, 1968.

SISSON, C.R. Aparelho urogenital do ruminante. In: GETTY, R. Anatomia dos animais domésticos. 5. ed. Rio de Janeiro: Interamericana, 1996. v.1, p.879-895.

UNANIAN, M.M.; SILVA, A.E.D.F.; MCMANUS, C. et al. Características Biométricas Testiculares para avaliação de touros zebuínos da Raça Nelore. Rev. Bras. Zootec., v.29, p.136-144, 2000.

WOLF, F.R.; ALMQUIST, J.O.; HALE, E.B. Prepuberal behaviour and puberal characteristics of beef bulls on high nutrient allowance. J. Anim. Sci., v. 24, p.761-765, 1965. 